\title{
¿Cómo debe ser el trabajo de Educación Física en Educación Infantil? ¿How should Physical Education work in Early Childhood Education be?
}

Víctor Arufe Giráldez

Universidad de A Coruña (España)

Resumen. La Educación Física en Educación Infantil concentra un gran protagonismo en el desarrollo óptimo de cada una de las esferas del niño: física, social, afectivo-emocional y psíquica. Dotar de una gran información y formación a los futuros docentes de Educación Infantil, enseñando los contenidos a tratar desde esta materia es una pieza clave en el puzle del currículo educativo. A través de este artículo se indaga en los contenidos de Educación Física que se deben abordar en las clases de Educación Infantil y cómo enseñarlos. Se expone también un modelo de ficha didáctica para el análisis de las tareas y juegos programados y un planteamiento de la sesión basada en siete momentos. Se concluye la importancia de realizar programas de Educación Física bien dirigidos y planificados para el bienestar físico, social y emocional del niño, la mejora de la adherencia al ejercicio físico y la importancia del juego como vehículo generador de aprendizaje, no solo de contenidos propios del área sino también de otras áreas temáticas.

Palabras clave. Educación Física; Educación Infantil; Planes de estudio; Currículo educativo; Psicomotricidad.

\begin{abstract}
Physical Education in Early Childhood Education focuses greatly on the optimal development of each of the children's spheres: physical, social, affective-emotional, and psychic. Providing future Early Childhood teachers with strong information and training while teaching them the contents that need to be developed in this subject is a cornerstone of the educational curriculum. This article investigates the Physical Education contents that should be worked on within Early Childhood Education, as well as how to teach them. It also presents a didactic sheet model for the analysis of tasks and planned games, as well as an approach to sessions based on seven moments. As a conclusion, carrying out well directed and planned Physical Education programs is essential for children's physical, social, and emotional well-being, and for increasing adherence to physical exercise. Moreover, games represent an important vehicle for generating learnings not only regarding area-specific contents, but also those from other thematic areas.
\end{abstract}

Key words. Physical Education; Early Childhood Education; Curriculum; Educational Curriculum; Psychomotricity.

\section{Introducción}

La formación de los futuros docentes en Educación Física en España ha quedado muy endeble tras el proceso establecido en Europa con la Declaración de Bolonia en 1999 (Espacio Europeo de Educación Superior, 1999), a partir de la cual se eliminó la Diplomatura en Educación Física y se crearon los Grado de Educación Infantil (con o sin Mención en Educación Física) y los Grados de Educación Primaria (con o sin Mención en Educación Física) regulados a través del Real Decreto 1393/2007 (Ministerio de Educación y Ciencia del Gobierno de España, 2007).

Posteriormente, la Agencia Nacional de Evaluación de la Calidad y Acreditación (ANECA) inició en España, el Programa de Convergencia Europea con la finalidad de integrar la Educación Superior en el Espacio Europeo de Educación Superior proponiendo una carga de 30 créditos de carácter optativo en los planes de formación de un título generalista o mención. A pesar de la no aceptación por parte de muchos colectivos de esta reducción de créditos, la propuesta siguió su cauce y se aprobó años más tarde justificando su convergencia con el resto de países de la Unión Europea (ArufeGiráldez, Lera-Navarro \& Varela-Garrote, 2009).

De esta forma, podemos comprobar como la formación de los futuros docentes en Educación Física de Educación Infantil y Educación Primaria depende inicialmente de las materias contempladas en los diferentes títulos de Grado de las universidades españolas. En este artículo haremos referencia a la formación de los futuros docentes de Educación Física desde el Grado de Educación Infantil.

\footnotetext{
Fecha recepción: 12-02-19. Fecha de aceptación: 08-09-19

Víctor Arufe Giráldez

varufe@yahoo.es
}

Algunos estudios que han abordado la formación vinculada a la Educación Física de los docentes, o futuros docentes, en Educación Infantil constatan que un número elevado de encuestados, aproximadamente un $83 \%$, consideran que la Administración debería contemplar la figura del especialista en psicomotricidad en esta etapa (Viscarro-Tomás, Antón-Rosera \& Cañabete-Ortiz, 2012). En otro trabajo realizado por Aguado-Ruíz (2014) se confirma la existencia de solamente dos materias obligatorias vinculadas a la Educación Física en los planes de estudio del Grado de Educación Infantil en la Universidad de Murcia con 6 créditos ECTS cada una de ellas.

Esta formación específica sobre Educación Física resulta insuficiente en la mayoría de los planes de estudio del título de Grado de Educación Infantil de las diferentes universidades españolas e incluso también en los planes de formación de los maestros de Educación Primaria con Mención en Educación Física (Dopico-Pedre, 2016; Gambau-I Pinasa, 2015).

Sugrañes-Justafré \& Ángel-Ferrer (2007) señalan que el desarrollo psicomotor del alumnado ha de estar contemplado en el proyecto de centro, debiendo ser los maestros los primeros responsables de la educación psicomotriz de los niños y siendo la figura de maestro especialista en psicomotricidad/Educación Física un refuerzo importante para esta área. En otro trabajo realizado en una muestra de 593 maestros de Educación Infantil, donde se preguntaba a los maestros acerca de su competencia formativa para realizar un proyecto de educación psicomotriz, la mayoría de los docentes reconocieron su falta de formación (MartínDomínguez \& Rodríguez-Sánchez, 2010).

En el caso de la Universidad de A Coruña, la formación vinculada a la Educación Física en el Grado de Educación Infantil suma un total de 15 créditos de los cuales solamente 6 son de carácter obligatorio. Existen actualmente un total de 
3 materias: Didáctica de la Expresión Corporal (6 créditos de carácter obligatorio), Juegos Motores (4.5 créditos de carácter optativo) Educación Psicomotriz y Habilidades Motrices Básicas (4.5 créditos de carácter optativo).

Es importante destacar la gran cantidad de términos utilizados por los diferentes autores a la hora de hablar de la Educación Física en Educación Infantil, términos como educación motriz, educación psicomotriz, educación física de base, expresión corporal, educación kinesiológica o predeporte (Gil-Madrona, Contreras-Jordán, González-Vílora \& Gómez-Barreto, 2008). Dado que el objetivo de este manuscrito no es el de aclarar las posibles diferencias y similitudes de cada uno de estos términos, se utilizarán como términos comunes el de Educación Física, como materia implantada en los currículos de Educación Infantil, Primaria y Secundaria/Bachiller y el término de competencia motriz para hacer referencia al nivel de logro alcanzado por el alumnado al cursar dicha materia. En ambos casos se entenderá la Educación Física como el trabajo del triángulo formado por el cuerpo, la mente y el medio donde el niño interactúa. Del mismo modo, se hará siempre referencia al movimiento corporal y las implicaciones que este trabajo puede ofrecer en los procesos cognitivos, afectivos-emocionales y relacionales en niños de 3 a 6 años.

Un reciente estudio sobre el concepto de Educación Física realizado en una muestra de 180 directores de centros educativos señala que esta materia está asociada al trabajo en 5 dimensiones: dimensión física, social, académica, espiritual y emocional (Araçilgar \& Cihan, 2018). Vinculado a la Educación Física está el concepto de competencia motriz, una competencia que se trabajaría con los contenidos propios de la Educación Física; buscando, a través de aprendizajes motrices, la adaptación a una multiplicidad de situaciones que poseen ciertas características comunes, consolidando automatismos en contextos estables o improvisando respuestas en contextos no estables. De esta forma, el niño debe decodificar e interpretar el entorno, descubrir los indicios reveladores y convertir la percepción en una capacidad de comprensión del sentido de la situación, el factor-clave de la competencia motriz (Pierre, 2018).

El presente manuscrito sintetiza toda la información necesaria para poder establecer una hoja de ruta de los contenidos que deben abordar los estudiantes universitarios del Grado de Educación Infantil, a fin de mejorar su competencia en el área de Educación Física y Psicomotricidad. Resaltando la importancia que tiene un correcto enfoque y dirección de estos contenidos para el desarrollo integral de los niños de tres a seis años. A su vez, este artículo sirve de guía para el profesorado de Educación Infantil que desee conocer en profundidad qué contenidos de Educación Física trabajar y cómo desarrollarlos en niños de tres a seis años.

\section{La Educación Infantil, la etapa educativa que más casti- ga el movimiento de los niños}

Es quizá en esta etapa donde más se castiga a la motricidad del niño, o a su competencia motriz, cuando estos debieran pasar más tiempo fuera del aula que dentro de ella. Los motivos son principalmente tres:

1) Porque no existe una formación sólida inicial que capacite a los futuros docentes con un gran conocimiento de la materia Educación Física, tal como se ha indicado en el anterior apartado.

2) Porque en muchas ocasiones el perfil del profesor que imparte docencia en los centros de Educación Infantil es muy diverso, desde profesorado con el Grado de Educación Infantil sin mención, a profesorado con mención en Educación Física o incluso profesorado de Educación Primaria que atiende a la etapa de Educación Infantil para impartir esta materia (Viscarro-Tomás, Antón-Rosera \& Cañabete-Ortiz, 2012; Martín-Domínguez \& Rodríguez-Sánchez, 2010).

3) El poco valor y reconocimiento del currículo educativo hacia el trabajo motriz de tres a seis años, planificando solamente una sesión a la semana el 55\% de los centros e incluso constatando en algunos estudios que cerca de un 17\% de centros educativos no imparten ninguna sesión de Educación Física en esta etapa (Pons \& Arufe-Giráldez, 2016).

Es importante aumentar el número de investigaciones orientadas a cuantificar la actividad física realizada en niños de tres a seis años, a fin de poder establecer líneas de trabajo en común y poder definir qué y cuánta actividad física es necesaria para poder crear adherencia a la práctica deportiva o hacia un estilo de vida saludable, o qué y cuánta es nece-

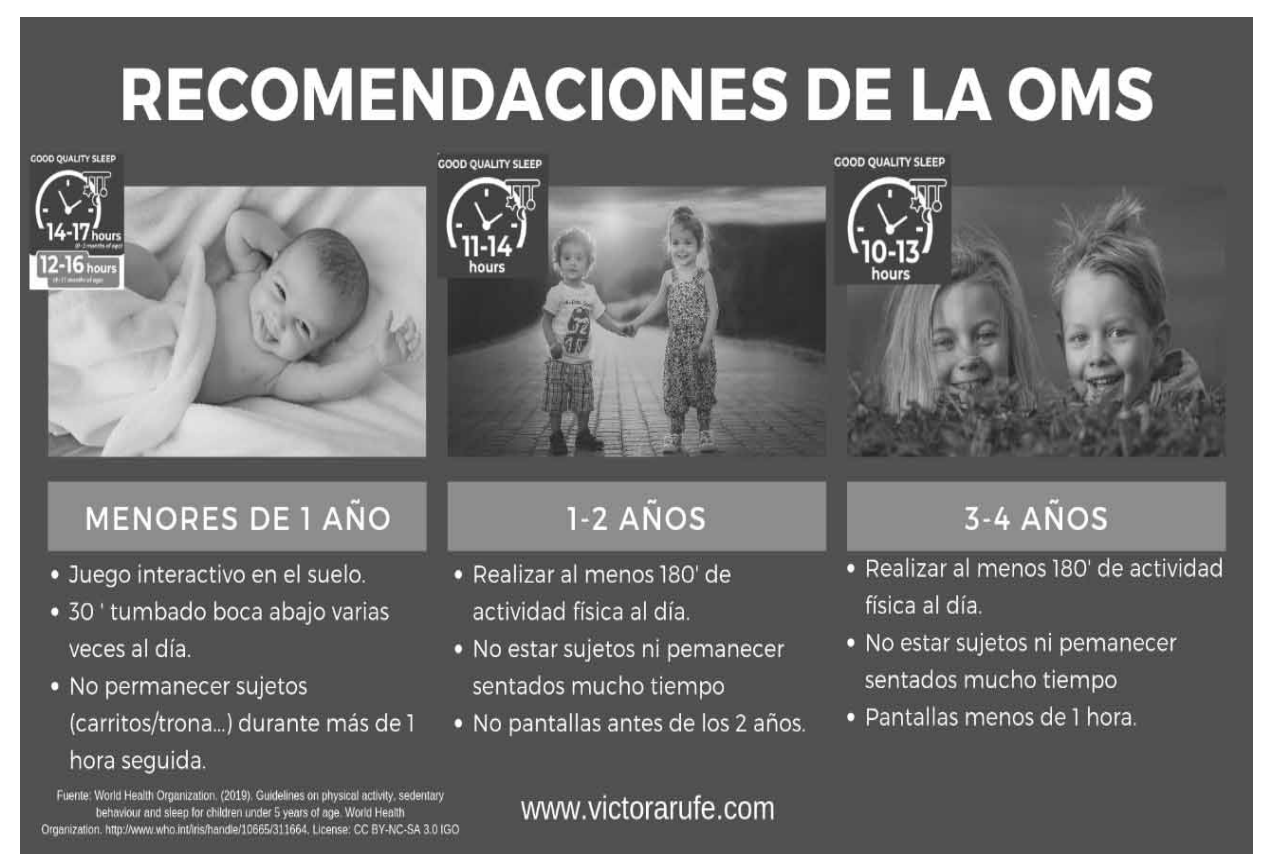

Figura 1. Recomendaciones de la Organización Mundial de la Salud destinadas a niños de 0 a 4 años. Fuente: Adaptada de World Health Organization (2019). 
saria para poder combatir el sedentarismo y prevenir patologías asociadas a este. Sabemos que programas de Educación Física bien dirigidos y planificados otorgan mejoras en el perfil psicomotor de los niños (Mas, Jiménez, \& Riera, 2018; Teixeira, Arufe-Giráldez, Abelairas-Gómez \& BarcalaFurelos, 2015). En etapas posteriores, algunos autores añaden que, además de programar correctamente las clases de Educación Física, se debe tener en cuenta la individualización de los juegos y ejercicios para conseguir más minutos de práctica de actividad física moderada-vigorosa en los estudiantes (Fairclough \& Stratton, 2005).

Ya sea por la escasez de trabajo de motricidad en la escuela o por la falta de educación hacia el movimiento desde las familias, la inactividad física en niños es una realidad. La Organización Mundial de la Salud ha emitido por primera vez en su historia un informe con recomendaciones de actividad física en niños de cero a cinco años, ante la alerta de la inactividad física mundial de este sector poblacional. En este documento señala la necesidad de que los niños de tres a cuatro años realicen al menos 180 minutos de actividad física al día, recomendando que no permanezcan sujetos ni sentados mucho tiempo y que tengan menos de una hora de pantallas al día (World Health Organization, 2019). Son unas recomendaciones importantes a las que la escuela debe dar respuesta con un mayor número de horas de Educación Física garantizando así la práctica motriz de los niños.

Algunas escuelas parecen estar concienciadas de la importancia del movimiento en niños de cortas edades. Así, en un estudio realizado con 1109 niños del condado rural de Sogn Fjordane, en el oeste de Noruega, con edades comprendidas entre los 2.7 y 6.5 años se detectaron unos niveles mayores de actividad física moderada-vigorosa en el centro educativo frente al hogar; resultando ser menos sedentarios en días de semana que los fines de semana (Nilsen, Anderssen, Resaland, Johannessen, Ylvisaaker \& Aadland, 2019). Los autores señalan que, aunque el ambiente en el hogar es un importante elemento para el bienestar de los niños, el marco escolar es fundamental para promover la actividad física estructurada y obtener así los mayores beneficios de esta práctica deportiva educativa, independientemente de las características de los niños y de los comportamientos de los padres, las actitudes, los recursos y factores socioeconómicos.

Se constata, por lo tanto, la necesidad de formar a los futuros maestros/as de Educación Infantil en el ámbito de la Educación Física, con el objetivo de que puedan potenciar este trabajo cuando estén incorporados a los centros educativos.

\section{¿Qué debe enseñarse al futuro profesorado de Educa- ción Infantil que imparta la materia de Educación Física o Psicomotricidad?}

La etapa de Educación Infantil contempla como dos de sus principios generales la necesidad de que todas las propuestas y experiencias de aprendizaje se aborden desde un enfoque integrado y globalizador; y que los métodos de trabajo en ambos ciclos se basen en las experiencias, en la actividad infantil y en el juego, aplicándose en un ambiente de seguridad, afecto y confianza para potenciar la autoestima y la integración social (Ministerio de Educación y Ciencia del Gobierno de España, 2008).

Observando estas directrices, es importante destacar que todos los contenidos a abordar en esta etapa incidan principalmente en las cuatro esferas del desarrollo humano: física, social, afectiva-emocional y psíquica.

Los contenidos elegidos por el profesorado universitario para enseñar al alumnado son importantes, pero todavía cobra una mayor relevancia el cómo se imparten, especialmente, en cómo experimentarlos con los niños. La metodología que apliquemos provocará un mayor aprovechamiento de los recursos del docente y de los contenidos en sí mismo. Especialmente, podrá garantizar uno de los grandes retos de la sociedad actual, promover la práctica deportiva o de ejercicio físico desde etapas inferiores para combatir el sedentarismo.

Es aquí donde introducimos el término de adherencia al ejercicio físico. Un término que implica la atención y el cuidado de numerosos factores, especialmente la motivación, tal como señalan en su artículo de revisión González-Calvo, Bores-García, Hortigüela \& Barba-Martín (2018) abordando los múltiples factores que influyen en la adherencia a los programas de ejercicio físico en los ámbitos educativos y extraescolar. Con la adherencia al ejercicio físico se consigue la generación de un estilo de vida saludable promoviendo el compromiso del niño con la práctica deportiva más allá del horario escolar. Sin lugar a dudas, un gran reto para los docentes de Educación Física, que, en el caso de Educación Infantil, tan solo cuentan con una hora a la semana para intentar motivar hacia la práctica deportiva a todo el alumnado.

\section{Sobre los contenidos a enseñar}

Los contenidos que se deben abordar en los planes de formación del profesorado de Educación Física en Educación Infantil serán los contenidos relacionados directamente con el trabajo del cuerpo, de la mente y del movimiento. La interacción cuerpo-mente es el principal objeto de estudio y trabajo en el ámbito de la Educación Física. El currículo de Educación Infantil del Gobierno de España señala tres grandes áreas de contenidos: Área de Conocimiento de sí mismo y autonomía personal, Área de Conocimiento del entorno y Área de lenguajes: comunicación y representación (Ministerio de Educación y Ciencia del Gobierno de España, 2008).

De la relación cuerpo-mente y su integración en el ambiente surgen comunicaciones intrapersonales e interpersonales:

- La comunicación intrapersonal hace referencia al entendimiento y escucha del cuerpo de uno mismo, a su manejo y dominio, a saber cuidarlo, respetarlo, aceptarlo, a la comunicación entre el cerebro y cada segmento corporal, a la creación de redes neuronales para mejorar el potencial motriz, a la mejora de las habilidades motrices básicas, de las capacidades físicas, de la expresividad corporal, pero también a las emociones y sensaciones y al desarrollo de las funciones cognitivas básicas y superiores.

- Mientras que la comunicación interpersonal exalta el valor del ser humano, entendido este como un integrante de la sociedad, en sus relaciones sociales, en la búsqueda de afecto, de socialización, empatía, cohesión grupal, respeto de las normas y adquisición de principios éticos y 
deontológicos. Es todo lo relacionado con los valores, con variables psicológicas y psicosociales, con la construcción de la sociedad, la ética y la moral, y con todo aquello donde el individuo no se encuentre solo, sino que se integre en un ecosistema social interactuando con otros seres, con el ambiente y formando parte de una estratificación social. También está incorporada aquí la función del lenguaje, constatando algunos autores la mejora de esta a través de una práctica psicomotriz bien dirigida (Rodríguez, Gómez, PrietoAyuso \& Gil-Madrona, 2017).

Los contenidos abordados deben integrarse también con un enfoque globalizador en las diferentes inteligencias múltiples del modelo propuesto por Howard Gardner (2011), haciendo hincapié en la inteligencia kinésico-corporal, intrapersonal e interpersonal, sin olvidar la lógico-matemática, visoespacial, musical, naturalista, lingüística o existencial, esta última todavía en estudio.

Pero la Educación Física también incide fuertemente en aspectos psicológicos y psicosociales. Diversos trabajos afirman que variables como la empatía, tolerancia, el autoconcepto, autoconfianza, motivación intrínseca o autoestima son potenciadas positivamente a través de la práctica de actividad física (Arufe-Giráldez, Zurita-Ortega, Padial-Ruz \& Castro Sánchez, 2019; González-Valero, ZuritaOrtega, Pérez-Cortés, Padial-Ruz, Ubago-Jiménez \& ChacónCuberos, 2019). Todos los contenidos expuestos en este apartado están respaldados por investigaciones clásicas realizadas en el ámbito de la psicología, psicomotricidad y fisiología, tales como Ajuriaguerra (1979), Gessell \& Amatruda (1972), Lapierre y Aucouturier (1977), Le Boulch(1972), Piaget (1975), Picq y Vayer (1969), Vaca y Varela (2008), Vygotsky (1979), Wallon (1969) entre otros.

Dicho esto, la enseñanza de la Educación Física en Educación Infantil en el alumnado universitario, futuros docentes de esta etapa, debe centrarse en trabajar los siguientes contenidos:

- Habilidades Motrices Básicas: Las habilidades motrices básicas se desarrollarán paralelamente al desarrollo psicomotor del niño, mejorando así las posibilidades de trabajo con su cuerpo. Se deben realizar juegos a través de los cuales se potencie y desarrollen las diferentes habilidades motrices, como la marcha, carrera, saltos, lanzamientos, rodamientos, giros, volteos, etc. Este trabajo establecerá mejoras en todas las esferas de su desarrollo y servirá de base para aprendizajes superiores. En este bloque se podrán trabajar reptaciones, manipulaciones de objetos y móviles, gateo, saltos, lanzamientos, giros, volteos, trepa, suspensiones, marcha, Figura 2. Contenidos a enseñar en Educación Física en Educación Infantil. Fuente: elaboración carrera...(Cechini-Estrada, propia.
Fernández-Losa \& Pallasá-Manteca, 2016; Gómez-Barreto, Gil-Madrona \& Contreras-Jordán, 2008; Andrés, 2014; Zubiaur-González, 2005). Pero también está contemplada aquí la iniciación a las habilidades gimnásticas, como la rueda lateral, el equilibrio invertido de brazos, voltereta atrás o adelante, entre otras.

- Componentes de la psicomotricidad o factores perceptivo motrices: Conjuntamente con las habilidades motrices podemos potenciar la competencia motriz trabajando diferentes elementos o factores que componen el trabajo psicomotriz. Nos referimos a la lateralidad, coordinación óculo manual, óculo podal, dinámico general, respiración, relajación, control tónico, equilibrio estático y dinámico, estructuración espacial, localización espacial, orientación espacial, estructuración temporal, estructuración espacio temporal, ritmo, percepción corporal, esquema corporal, enres. El desarrollo armónico de estos configurará a un niño capaz de adquirir una mayor riqueza y destreza motriz, al conocer más profundamente el comportamiento de su cuerpo (Ibáñez-Gericke, 2018; Mérida-Serrano, de los Ángeles Olivares-García, \& Gonzalez-Alfaya, 2018; Pérez-González, 2011; Silva, Abilleira \& Gradín, 2018). Se incluyen aquí también contenidos vinculados a la expresión corporal, dramatización, cuentos motores, narrativa corporal creativa (NCC), teatro, mimo y pantomima, danza, bailes, Finger Tutting y actividades con soporte musical, así como actividades y técnicas diversas de relajación y respiración.

- Capacidades físicas: Para la mejora de la condición física y consecuentemente de la salud del niño es necesario trabajar también en los juegos las diferentes capacidades físicas: fuerza, resistencia, velocidad, flexibilidad y agilidad. También sus manifestaciones derivadas, fuerza resistencia, velocidad de desplazamiento, velocidad gestual, velocidad de reacción, resistencia aeróbica o resistencia aláctica (Arriscado, Dalmau, Zabala \& Muros, 2017; Latorre-Román, Moratre otros. El trabajo y desarrollo de cada uno de los factores psicomotrices favorecerá el aprendizaje de técnicas superio-

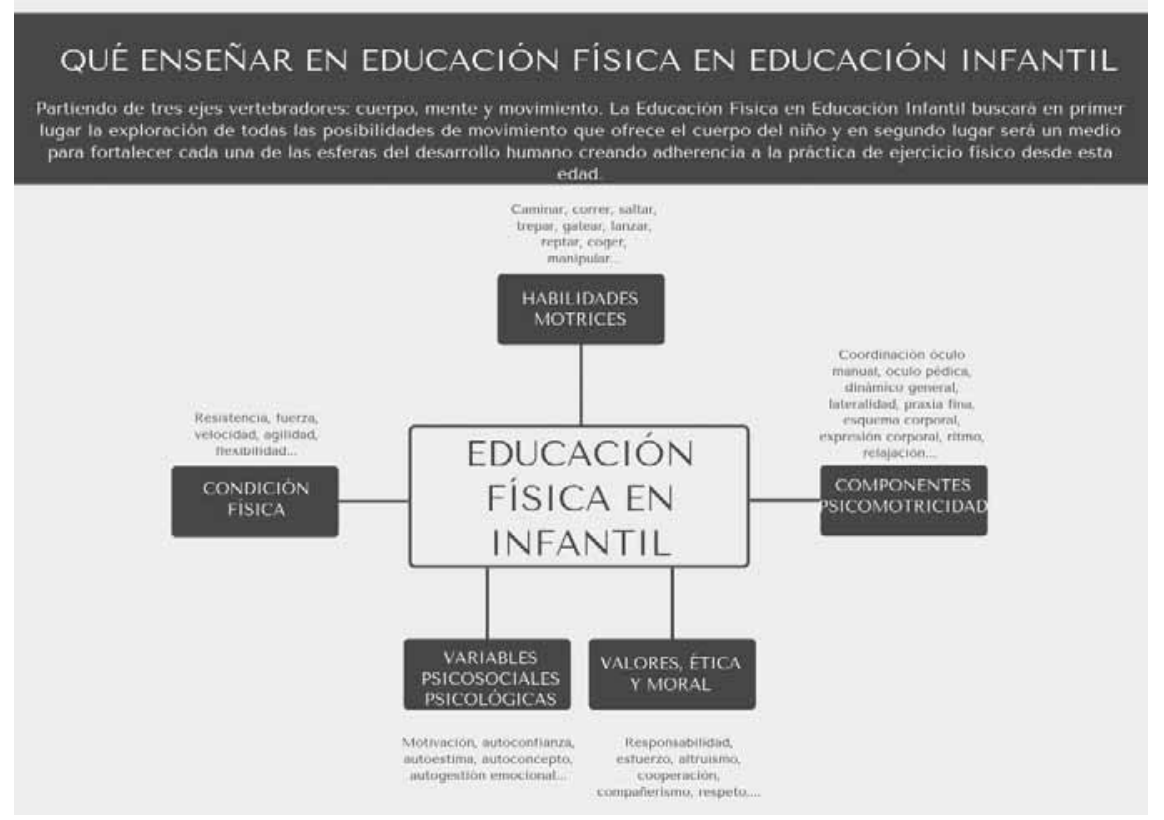


López, Fernández-Sánchez, Sala-Sánchez, Moriana-Coronas \& García-Pinillos, 2015; López Mora, 2017; Secchi, García, \& Arcuri, 2016).

- Variables psico-sociales: Comentábamos anteriormente la importancia de trabajar todo lo relacionado con la mente, en este caso, hablamos de trabajo de la autoestima, motivación intrínseca, autoconfianza, autocontrol, autogestión emocional, reconocimiento de las emociones, locus de control interno o situaciones estresantes. La propia práctica motriz potenciará implícitamente el autoconcepto. El niño podrá conocer su cuerpo y sus posibilidades, aumentando posteriormente su autoestima. Reconocerá las fortalezas que tiene, intentará afrontar retos con su propio dominio corporal y favoreciendo, poco a poco, una mayor autoconfianza, llegando a obtener la necesaria autonomía para su día a día (Martinek, Holland \& Seo, 2019; MartínezMartínez \& González-Hernández, 2018; Missana, Rajhans, Atkinson \& Grossmann, 2014; Sampaio, De David, Filho \& Húngaro, 2017).

- Valores, ética y moral: Como individuos que forman parte de una sociedad, los niños deben adquirir también grandes valores que permitan realzar su capital humano y elevarlo a la excelencia de calidez y calidad humana. Trabajar valores a través de la práctica motriz es uno de los grandes objetivos de los docentes, potenciando así el altruismo, la empatía, la cohesión grupal, compañerismo, cooperación, saber ganar/saber perder, resiliencia, frustración, respeto de la naturaleza, respeto a los animales, liderazgo, competitividad, esfuerzo, juego limpio, etc. así como el trabajo de modelos basados en la responsabilidad social y personal a través de la Educación Física (Fernández-Gavira, Jiménez-Sánchez \& Fernández-Truan, 2018; Menéndez Santurio, 2018; Monzonís-Martínez, 2019; Prat, Camerino, Castañer, Andueza \& Puigarnau, 2019)

Existen otros dos grandes bloques que en esta etapa no se contempla su trabajo, debido principalmente a la propia inmadurez del cerebro de los niños que todavía no está preparado y receptivo para aprendizajes superiores. Pero este trabajo sí que está presente en etapas como en Educación Primaria o Educación Secundaria. Estamos refiriéndonos al bloque de aprendizaje técnico, aprendizaje de la técnica de diferentes deportes y al bloque del aprendizaje táctico, referido al aprendizaje de sistemas tácticos individuales y colectivos para la enseñanza de diferentes deportes.

\section{Sobre la metodología a utilizar}

El Currículo de Educación Infantil (Ministerio de Educación y Ciencia del Gobierno de España, 2008) expone unos principios metodológicos a tener en cuenta en esta etapa.

Primeramente, destaca la atención a la diversidad, atendiendo a los diferentes periodos de desarrollo de los niños y ofreciendo respuestas adecuadas a las necesidades de cada niño. En segundo lugar, apuesta por el enfoque globalizador otorgando a los niños un aprendizaje desde una perspectiva integrada y diversa.

El aprendizaje significativo es otro de los principios que debe primar en esta etapa. No solo será suficiente con enseñar contenidos, sino que el niño deberá ser consciente de ese aprendizaje, saber por qué, para qué, cómo, y otras cuestiones que se pueden plantear en relación con el aprendizaje y su aplicación en el día a día.
El juego, la experimentación y la actividad práctica deben constituir el vehículo principal para la enseñanza de todos los contenidos. También el espacio, los materiales y el tiempo deben planificarse de forma correcta y especialmente crear espacios que generen aprendizaje. La propia arquitectura del aula es también una gran fuente educativa, dado que los espacios también educan, destacando el importante papel desarrollado por los rincones de aprendizaje donde se puedan trabajar diferentes contenidos y habilidades previamente planificados por el docente.

Por último, el currículo también destaca la necesidad de que esta etapa y todo lo que se genere en ella sea compartida por los profesionales de la educación y por las familias. Los centros de Educación Infantil deben ser espacios para la convivencia y estar abiertos al diálogo continuo con las familias.

Son estos, por tanto, unos principios metodológicos a tener en cuenta. Para cumplirlos no existe una metodología milagro que ofrezca todas las necesidades del docente. Lo importante es variar los métodos de enseñanza y especialmente conocer las implicaciones que conlleva aplicar uno u otro. En el caso de la etapa de Educación Infantil lo fundamental es utilizar metodologías activas que busquen la participación del alumnado, que impliquen al niño en todo el proceso y que éste sea más valorado que el propio resultado. En un estudio realizado sobre el aprendizaje activo para el desarrollo de la psicomotricidad y el trabajo en equipo se constató, luego de la intervención didáctica, una mayor valoración positiva del alumnado en el desempeño de diversas competencias (Aristizabal-Almanza, Ramos-Monobe \& ChirinoBarceló, 2017). Sentirse parte de un equipo de trabajo repercutió positivamente en los objetivos propuestos, que al realizar de manera constante una reflexión individual sobre sus prácticas, fueron capaces de identificar aciertos y desaciertos para construir conocimiento en la interacción con las demás personas lográndose que el alumnado fuera más responsable y autodirigido. Recomendando los autores de este trabajo proveer al personal docente de más experiencias de aprendizaje basadas en principios de aprendizaje activo.

Las metodologías basadas en la resolución de problemas, descubrimiento guiado, enseñanza recíproca o enseñanza en microgrupos en Educación Infantil son muy efectivas y poseen una alta gama de beneficios para el desarrollo social, afectivo-emocional y psíquico del niño. Las actividades por parejas, tríos y/o grupos son también un potenciador de las habilidades sociales en los niños (Gil-Madrona, Gutiérrez-Marín \& Madrid-López, 2012).

Pero también podemos buscar métodos de enseñanza basados en el mando directo ante situaciones que puedan implicar un potencial riesgo para la integridad física del alumno. En esta situación, le diremos cómo hacer la tarea y dónde debe prestar atención para evitar un posible daño. Tal es el caso de trabajar habilidades de escalada o trepa en las espalderas, asumiendo el potencial peligro de caída que puede tener el niño durante el juego sobre estos elementos.

El diálogo constante con los niños debe formar parte de cualquier metodología docente, facilitando la comunicación de las continuas dudas que puedan tener durante la realización de las tareas o juegos y conociendo su estado de bienestar y conformidad con los ejercicios realizados, así como 
con los integrantes del grupo clase.

La neuroeducación, o el conocimiento sobre cómo aprende el cerebro, ha entrado sólidamente en los programas de actualización y reciclaje de la formación docente en los últimos cinco años. Sin embargo, los planes de estudio de formación del profesorado todavía no contemplan firmemente los hallazgos beneficiosos para la docencia de la aplicación de los principios de la neuroeducación en el aula. Uno de los principales expertos en neuroeducación en España, el profesor Francisco Mora (2014) destaca la experimentación, la curiosidad, el interés, la práctica de actividad física y las emociones como grandes explosivos que despiertan la atención del alumno y mejoran los procesos cognitivos implicados en el aprendizaje. En el ámbito de la Educación Física y la neurociencia destacan las aportaciones de Pellicer-Royo, López-González, Mateu-Serra, Mestres-Pastor, Meritxell \& Ruíz-Omeñaca(2015).

Nuevamente observamos, como la actividad física es uno de los pilares que debe acaparar la atención en los centros de Educación Infantil. El juego motor se convierte así en un vehículo ideal para incorporar los contenidos del currículo de Educación Infantil con un enfoque globalizador, pudiendo integrar nociones matemáticas y actividad física, o valores, ciencias naturales o cualquier otro contenido del currículo, pues son todos ellos canalizables a través de la Educación Física.

Pero también es necesario el juego libre, el juego que se enfrenta al juego dirigido, un juego en el cual el niño pone sus propias reglas, el tiempo de inicio y el fin de cada juego creado, y pulsa el botón de arranque del mecanismo de la creatividad. Gray (2015) demuestra, basándose en pruebas desde la antropología, la psicología y la historia, que el juego libre es el medio principal por el cual los niños aprenden a controlar sus vidas, a resolver problemas, a llevarse bien con sus compañeros y a ser emocionalmente resistentes, indicando el autor que este tipo de juego está en declive y que la escuela ha de potenciarlo.

\section{La sesión de Educación Física. Partes y ambientes}

Existen múltiples formas de organizar en el tiempo y en el espacio las sesiones de Educación Física. En este apartado proponemos una de ellas, pero es importante destacar que al margen de esta estructuración, lo más importante de una sesión de psicomotricidad o Educación Física es aprovechar al máximo el tiempo disponible para aumentar el tiempo de compromiso motor, que es el tiempo en el cual el alumno está en movimiento.

En esta línea, algunos autores señalan unas recomendaciones para los docentes, tales como plantear actividades, juegos o deportes que sean motivadores para los niños, realizar las actividades iniciales por parejas o por tríos, ya que de esta manera se conseguirá que los niños obtengan un mayor rendimiento o evitar juegos en los que los niños queden sentados mientras otros ejecutan (Aguado-Sanchís

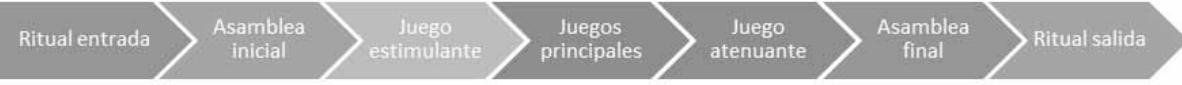

Figura 3. Modelo de sesión de Educación Física basada en 7 momentos. Fuente: elaboración propia.
\& Mateo-Ortega, 2018). En la figura 3 se presenta un modelo de sesión de Educación Física basado en siete momentos.

El primer momento consiste en un pequeño ritual de entrada a través del cual se marque el inicio de las sesiones de psicomotricidad. Se trata de un grito de activación en conjunto y una estimulación hacia la práctica de actividad física. Se invita a los niños a formar una rueda y poner sus puños juntos al tiempo que gritan «iAquí venimos aquí estamos para pasarlo como enanos!». Es un momento de unión y cohesión donde todos los alumnos tendrán un fin en común: la diversión.

Posteriormente a este momento, iniciaríamos una fase de asamblea donde explicamos brevemente a los niños qué actividades haremos en la sesión, o empezaremos a contarles una historia como hilo conductor de todos los juegos y despertando la curiosidad de los niños. Las sesiones pueden ser monotemáticas o multitemáticas, abordando numerosos contenidos de la Educación Física.

Tras esta pequeña asamblea se iniciará un juego estimulante o de calentamiento. Es una actividad que debe preparar para los contenidos a trabajar en los juegos principales. Esta no debe ser ni muy estática ni tampoco muy activa, y estará vinculada a la temática de la sesión. Posteriormente se desarrollarán los juegos principales destinados al trabajo de los contenidos que estuviesen planificados en la sesión. Podemos optar por sesiones monotemáticas, por ejemplo, de trabajo con especial incidencia en una o dos habilidades o capacidades, o sesiones multitemáticas con incidencia en múltiples contenidos de la psicomotricidad. Quizá este modelo sea más rico y productivo para los niños, dejando el otro para cubrir necesidades educativas o de refuerzo en algún contenido que pudiese estar debilitado en el alumnado.

Una vez realizada la parte principal empezaremos con el juego atenuante, un juego que busca devolver a los niños el estado de energía inicial de cara a que puedan acudir a otra clase con los niveles de atención equilibrados y totalmente despiertos. Estos juegos atenuantes suelen aprovecharse para el trabajo de contenidos que requieran algo menos de movilidad corporal, tales como: relajación, expresión corporal, mimo, discriminación auditiva, masaje o trabajo de respiración.

Posteriormente realizaremos una breve asamblea final invitando a los niños a comentar qué juego les gustó más, dónde se sintieron más cómodos, qué dificultades tuvieron, si disfrutaron, a qué juego les gustaría volver a jugar, etc. Se trata de que todos los niños hablen y expongan sus sentimientos y emociones. Por último, haremos un ritual de sali$d a$, formando nuevamente una rueda, pero esta vez abrazados y diciendo «Y con esta actividad más relajada me voy para clase de forma calmada».

\section{Modelo de ficha didáctica para el docente}

Tal como se ha indicado anteriormente, en cada juego y en cada actividad realizada en Educación Física en Educación Infantil se debe involucrar a las diferentes esferas del desarrollo humano: social, afectivo-emocional, psíquica y física. Esta es la ri- 


\begin{tabular}{|c|c|c|c|c|c|}
\hline \multicolumn{6}{|c|}{$\begin{array}{l}\text { Edades: } \square 0-1 \quad \square 1-2 \quad \square 2-3 \quad \square 3-4 \quad \square 4-5 \quad \square 5-6 \\
\text { Área de conocimiento y Bloque de contenido del Currículo }\end{array}$} \\
\hline \multicolumn{2}{|c|}{ AREA DE CONOCIMIENTO } & \multicolumn{2}{|c|}{$1^{\circ}$ CICLO CONTENIDOS } & \multicolumn{2}{|c|}{$2^{\circ} \mathrm{CICLO}$ CONTENIDOS } \\
\hline \multicolumn{2}{|c|}{$\begin{array}{l}\text { पConocimiento de sí mismo y } \\
\text { autonomía personal }\end{array}$} & \multicolumn{2}{|c|}{$\begin{array}{l}\text { QEl cuerpo } \\
\text { QLa identidad, la autoestima y las } \\
\text { emociones } \\
\square \text { El cuidado personal }\end{array}$} & \multicolumn{2}{|c|}{$\begin{array}{l}\text { QEl cuerpo y la propia imagen } \\
\text { QJuego y movimiento } \\
\text { QLa actividad cotidiana } \\
\text { QCuidado personal y salud }\end{array}$} \\
\hline \multicolumn{2}{|c|}{ Q Conocimiento del entorno } & \multicolumn{2}{|c|}{$\begin{array}{l}\text { QEl medio físico } \\
\text { QAcercamiento a la naturaleza } \\
\text { QCultura y vida en sociedad }\end{array}$} & \multicolumn{2}{|c|}{$\begin{array}{l}\text { QMedio físico: elementos, } \\
\text { relaciones y medida } \\
\text { QAcercamiento a la naturaleza } \\
\text { QCultura y vida en sociedad }\end{array}$} \\
\hline $\begin{array}{l}\text { QLenguajes: Comun } \\
\text { representación }\end{array}$ & icación & \multicolumn{2}{|c|}{$\begin{array}{l}\text { QComunicación gestual y oral } \\
\text { QExpresión plástica y musical } \\
\text { QRepresentación escrita y dramática }\end{array}$} & \multicolumn{2}{|c|}{$\begin{array}{l}\text { QLengua verbal } \\
\text { QLenguaje artística: } \\
\text { musical-corporal } \\
\text { QLenguaje audiovisual }\end{array}$} \\
\hline \multicolumn{6}{|l|}{$\begin{array}{l}\text { Material: } \\
\text { Objetivos }\end{array}$} \\
\hline Psicomotricidad & \multicolumn{3}{|c|}{$\begin{array}{l}\text { Valores, Variables p sicológicas, } \\
\text { educativas y sociales }\end{array}$} & $\begin{array}{l}\text { Habilidades } \\
\text { Motrices }\end{array}$ & $\begin{array}{l}\text { Capacidades } \\
\text { Físicas }\end{array}$ \\
\hline $\begin{array}{l}\text { Apreciación } \\
\text { trayectorias } \\
\text { Coordinación óculo } \\
\text { manual } \\
\text { Coordinación óculo } \\
\text { pédica } \\
\text { Coordinación } \\
\text { dinámica general } \\
\text { Estructuración/percep } \\
\text { ción espacial } \\
\text { Estructuración/percep } \\
\text { ción temporal } \\
\text { Equilibrio dinámico } \\
\text { Equilibrio estático } \\
\text { Esquema/percepción } \\
\text { corporal } \\
\text { Expresión corporal } \\
\text { Lateralidad } \\
\text { Praxia fina } \\
\text { Praxia gruesa } \\
\text { Relajación/ } \\
\text { respiración } \\
\text { Ritmo } \\
\text { Tonicidad/control } \\
\text { postural }\end{array}$ & $\begin{array}{l}\text { Aceptación } \\
\text { Autonomía } \\
\text { Autoestima } \\
\text { anza } \\
\text { Creatividac } \\
\text { Constancia } \\
\text { Cooperació } \\
\text { erismo } \\
\text { Disciplina } \\
\text { Empatía } \\
\text { Esfuerzo } \\
\text { Estrategia/t } \\
\text { Educación } \\
\text { Educación } \\
\text { Educación } \\
\text { Educación } \\
\text { Generosida } \\
\text { Hábitos sal } \\
\text { Habilidade } \\
\text { comunicati }\end{array}$ & $\begin{array}{l}\text { reglas } \\
\text { autoconfi } \\
\text { fantasía } \\
\text { /compañ } \\
\text { ctico } \\
\text { mocional } \\
\text { ial } \\
\text { ara la paz } \\
\text { utricional } \\
\text { dables }\end{array}$ & $\begin{array}{l}\text { Honestidad } \\
\text { Integración/acepta } \\
\text { ción } \\
\text { Igualdad de } \\
\text { género } \\
\text { Juego limpio } \\
\text { Liderazgo } \\
\text { Responsabilidad } \\
\text { Roles } \\
\text { Resiliencia } \\
\text { Respeto } \\
\text { Respeto medio } \\
\text { ambiente } \\
\text { Saber } \\
\text { ganar/perder } \\
\text { Solidaridad } \\
\text { Socialización } \\
\text { Superación } \\
\text { Educación } \\
\text { sensorial } \\
\text { Educación } \\
\text { artística }\end{array}$ & $\begin{array}{l}\text { Desplazamientos } \\
\text { Giros } \\
\text { Manipulación } \\
\text { Lanzamientos } \\
\text { Recepciones } \\
\text { Rodamientos } \\
\text { Saltos } \\
\text { Suspensión/ } \\
\text { tracción } \\
\text { Transportes }\end{array}$ & $\begin{array}{l}\text { Agilidad } \\
\text { Flexibilidad } \\
\text { Fuerza } \\
\text { Resistencia } \\
\text { Velocidad }\end{array}$ \\
\hline \multicolumn{6}{|c|}{ Procesamiento de la Información } \\
\hline $\begin{array}{c}\text { Tarea } \\
\text { predominantemente } \\
\text { perceptiva }\end{array}$ & $\begin{array}{r}\text { Ta } \\
\text { predomin } \\
\text { deci }\end{array}$ & emente & Tarea pre & dominantemente $\mathrm{e}$ & utiva \\
\hline \multicolumn{6}{|c|}{$\begin{array}{l}\text { Representación gráfica: } \\
\text { Observaciones para el docente: } \\
\text { Organización inicial: } \\
\text { Desarrollo: } \\
\text { Variantes: }\end{array}$} \\
\hline
\end{tabular}

Figura 4. Modelo de ficha didáctica global para la elaboración de juegos y tareas motrices en Educación Infantil. Fuente: Elaboración propia.

queza de la Educación Infantil, trabajar con el cuerpo del niño desde un punto vista global en el que nos alejemos de tareas repetitivas y mecánicas o con una secuencia de movimientos prefijada y hermética.

De esta forma, cada actividad propuesta en el aula debe afectar a diversos universos del niño y así se debe planificar y programar por parte del docente. En la figura 4 se expone un modelo de análisis didáctico de cada juego con el consiguiente registro de los contenidos y objetivos adaptados al Currículo de Educación Infantil del Ministerio de Educación y Formación Profesional del Gobierno de España (Ministerio de Educación y Ciencia del Gobierno de España, 2008), y que puede ser utilizado para cualquier propuesta realizada para el aula de Educación Infantil.

En la ficha, en primer lugar, se indica el nombre del juego. Debe ser un nombre atractivo y que invite a jugar a los niños. Posteriormente se señalan las edades a las que está destinado, siempre teniendo en cuenta la posible variabilidad en cuanto a maduración de los niños con una misma edad cronológica. Se incluye también un apartado para hacer referencia a los bloques $y$ áreas donde se integra ese juego y termina esta sección con la descripción del material utilizado, que debe ser siempre cuantificado y detallado.

En la segunda parte de la ficha figura su columna vertebral, los cinco grandes bloques que comentamos anteriormente y que enriquecen y aportan a cada una de las esferas del desarrollo humano de los niños. Los bloques correspondientes a las variables psicológicas y psicosociales se han unificado y a mayores se integra un apartado donde el docente debe indicar qué mecanismo del procesamiento de la información tiene más protagonismo en ese juego basándonos en el modelo de desarrollo del control motor a partir de teorías del procesamiento de la información (Marteniuk, 1976). Es importante este análisis dada la creciente red de conexiones neuronales en esta franja etaria y poder analizar así las tareas que demanden una mayor atención al mecanismo perceptivo, frente al decisivo o ejecutivo. Esta clasificación implica también el conocimiento del mundo sensorial, a través de las sensaciones interoceptivas, propioceptivas y exteroceptivas. Estas son de vital relevancia para explicar cómo el cerebro recopila la información del entorno y del propio cuerpo, la codifica, toma una decisión y ejecuta una respuesta verbal, fisiológica o motora.

Por último, se incluyen las partes comunes a otros modelos de fichas de juegos, en las que el docente incorporará información sobre la organización inicial del juego, desarrollo del mismo, posibles variantes y observaciones a destacar para la buena ejecución de la actividad.

\section{Conclusiones}

La etapa de Educación Infantil es una etapa de gran importancia para el desarrollo físico, social, afectivo, psíquico y emocional de los niños. La Educación Física bien dirigida y planificada puede contribuir mucho al desarrollo de estos 
universos. Además, es un buen medio para generar aprendizaje en otras áreas temáticas o campos de conocimiento, como el lenguaje, matemáticas, ciencias naturales o idiomas, entre otros. El juego se convierte como un excelente medio de conocimiento del mundo personal y del mundo que rodea a los niños, incluidos los compañeros.

Se han descrito los contenidos más importantes que deben enseñarse en esta franja etaria, apostando por una mayor cantidad de tiempo de compromiso motor y especialmente demandando una mayor cantidad de horas de Educación Física planificadas en el currículo educativo.

Se expusieron las líneas metodológicas a tener en cuenta por los docentes, buscando metodologías activas que impliquen la participación de los niños. Se presentó también un modelo de sesión basado en siete momentos, a través de los cuales se abordan y se potencian los diferentes bloques de contenidos a trabajar en esta etapa.

Por último, se presentó un modelo de ficha de análisis didáctico global de los juegos y tareas a realizar en Educación Física en Educación Infantil, desglosando los contenidos abordados en base a las áreas de conocimiento del currículo educativo y cuantificando los contenidos involucrados en cada una de las actividades a realizar.

El presente artículo ha pretendido contribuir al esclarecimiento de los contenidos que se deben enseñar al futuro profesorado de Educación Infantil resaltando la importancia que desempeña el correcto trabajo de Educación Física en niños de tres a seis años principalmente. Este manuscrito también sirve de orientación para el profesorado en activo que ejerce su docencia en Educación Física en el ámbito de la Educación Infantil. Como líneas futuras de investigación se propone seguir investigando en cada uno de los bloques de contenidos a potenciar desde la Educación Física a través de estudios longitudinales y transversales, así como revisiones de literatura y estudios empíricos. Aunque este trabajo se ha centrado en la franja etaria de tres a seis años, se ha detectado en la revisión de literatura para su confección un escaso número de trabajos que aborden la otra etapa de educación infantil, de cero a tres años. Como líneas futuras de investigación son necesarias más aportaciones que sigan demostrando los beneficios del trabajo corporal desde el nacimiento hasta los tres años a través de investigaciones de corte cuantitativo y cualitativo.

\section{Referencias}

Aguado-Ruíz, F. (2014). Psicomotricidad: formación de los maestros de Educación infantil y educación física. En P. Miralles-Martínez,

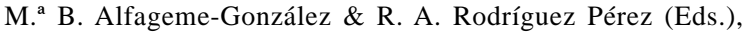
Investigación e Innovación en Educación Infantil. (273-282). Murcia, España: Universidad de Murcia.

Aguado-Sanchis, E. \& Mateo-Ortega, A. (2018). Los tiempos en la sesión de Educación Física en Educación Primaria: análisis y propuestas. Universitat Jaume I. Retos: Nuevas Tendencias en Educación Física, Deporte y Recreación, (35), 126-129. https:/ /doi.org/10.24215/23142561e050

Ajuriaguerra, J. (1979). Manual de psiquiatría infantil. Barcelona: Toray-Masson

Araçilgar, E. \& Cihan, B. B. (2018). Metaphoric perceptions of school principals towards physical education term. International Journal of Higher Education, 7(5), 194-205. https://doi.org/ 10.5430/ijhe.v7n5p194

Aristizabal-Almanza, J. L., Ramos-Monobe, A. \& Chirino-Barceló, V. (2017). Aprendizaje activo para el desarrollo de la psicomotricidad y el trabajo en equipo. Revista Electrónica Educare, 22(1), 1. https://doi.org/10.15359/ree.22-1.16

Arriscado, D., Dalmau, J. M., Zabala, M. \& Muros, J. J. (2017). Valores de condición física en escolares del norte de España. Journal of Sport and Health Research, 9(2), 211-222.

Arufe-Giráldez, V., Lera-Navarro, A. \& Varela-Garrote, L. (2009). La mención de Educación Física en el título de Grado de Educación Primaria; En Arufe-Giráldez. A.; Lera-Navarro, A.; Fraguela-Vale, R. \& Varela-Garrote, L. (Eds.), La Educación Física en la sociedad actual ( $1^{\circ}$ ed., pp. 13-30). Sevilla: Wanceulen. https://doi.org/ 10.17979/sportis.2017.3.1.1944

Arufe-Giráldez, V., Zurita-Ortega, F., Padial-Ruz, R. \& Castro-Sánchez, M. Association between Level of Empathy, Attitude towards Physical Education and Victimization in Adolescents: A MultiGroup Structural Equation Analysis. Int. J. Environ. Res. Public Health 2019, 16, 2360.

Cechini-Estrada, J. A., Fernández-Losa, J. L. \& Pallasá-Manteca, M. (2016). La precisión del movimiento imaginado y la recepción de balón en niños. Revista Internacional de Medicina y Ciencias de la Actividad Física y del Deporte, (62), 297-315. https:// doi.org/10.15366/rimcafd2016.62.008

Dopico-Pedre, M. (2016). Estudio descriptivo sobre la formación inicial de los/as maestros y maestras de Educación Física escolar. Sportis Sci J, 2(2), 188 205. http://dx.doi.org/10.17979/ sportis.2016.2.2.1429

Espacio Europeo de Educación Superior. (1999). Declaración de bolonia. Retrieved from http://www.eees.es/es/eees-desarrollocronologico. https://doi.org/10.4272/978-84-9745-387-5.ch9

Fairclough, S. \& Stratton, G. (2005). Physical activity levels in middle and high school physical education: A review. Pediatric Exercise Science, 17(3), 217-236. https://doi.org/10.1123/pes.17.3.217

Fernández-Gavira, J., Jiménez-Sánchez, M. B. \& Fernández-Truan, J. C. (2018). Deporte e inclusión social: Aplicación del programa de responsabilidad personal y social en adolescentes. Revista de Humanidades, (34), 39-58. https://doi.org/10.5944/ rdh.34.2018.19209

Gambau-I Pinasa, V. (2015). Las problemáticas actuales de la educación física y el deporte escolar en España. Revista Española de Educación Física y Deportes, 411, 53-69.

Gardner, H. (2011). Frames of mind: The theory of multiple intelligences. New York: Basic Books.

Gesell, A. \& Amatruda, C. (1945). Diagnóstico del desarrollo normal y anormal del niño: métodos clínicos y aplicaciones prácticas (B. Serebrinsky, Trad.). Buenos Aires: Paidós. (Original publicado en 1941).

Gil-Madrona, P., Contreras-Jordán, O. R., González-Víllora, S. \& Gómez-Barreto, I. M. (2008). Justificación de la Educación Física en la educación infantil. Educación y Educadores, 11(2), 159177. https://doi.org/10.1174/021037008784132950

Gil-Madrona, P., Gutiérrez-Marín, E. C. \& Madrid-López, P. D. (2012). Incremento de las habilidades sociales a través de la expresión corporal: la experiencia en clases de iniciación al baile. Cuadernos de Psicología del Deporte, 12(1). https://doi.org/ $10.6018 / 249131$

Gómez-Barreto, I. M., Gil-Madrona, P. \& Contreras-Jordán, O. R. (2008). Habilidades motrices en la infancia y su desarrollo desde una educación física animada. Revista Iberoamericana de Educación, 47(47), 71-96. https://doi.org/10.1174/ 021037008784132950

González-Calvo, G., Bores-García, D., Hortigüela, D. \& Barba-Martín, R. (2018). Adherencia a un programa de ejercicio físico en los ámbitos educativos y extraescolar. Apunts, (134), 39-54. http:// dx.doi.org/10.5672/apunts.2014-0983.es.(2018/4).134.03

González-Valero, G., Zurita-Ortega, F., Pérez-Cortés, A., Padial-Ruz, R., Ubago-Jiménez, J. \& Chacón-Cuberos, R. (2019). Estudio descriptivo del clima motivacional percibido hacia el deporte según el sexo de los futuros docentes de Educación Física. Sportis. Scientific Journal of School Sport, Physical Education and Psychomotricity, 5(1), 85-100. https://doi.org/10.17979/ sportis.2019.5.1.3479

Gray, P. (2015). Free to learn. New York. USA: Ingram Publisher Services.

Ibáñez-Gericke, T. (2018). Música e improvisación en 


\section{3}

Lapierre, A. \& Aucouturier, B. (1977). Educación vivenciada. Asociación de contrastes, estructuras y ritmos. Barcelona: Científico-Médica.

Le Boulch, J. (1972). La educación por el movimiento en edad escolar. Buenos Aires: Paidós.

Latorre-Román, A., Mora-López, D., Fernández-Sánchez, M., SalaSánchez, J., Moriana-Coronas, F. \& García-Pinillos, F. (2015). Test-retest reliability of a field-based physical fitness assessment for children aged 3-6 years. Nutrición Hospitalaria: Órgano Oficial De La Sociedad Española de Nutrición Parenteral y Enteral, 32(4 (Octubre 2015)), 1683-1688.

López-Mora, D. (2017). Evaluación y desarrollo de la condición física en niños preescolares. Universidad de Jaén.

Marteniuk, R. (1976). Information processing in motor skills. United States: Harcourt College Publishers.

Martinek, T., Holland, B. \& Seo, G. (2019). Understanding physical activity engagement in students: Skills, values, and hope. RICYDE. Revista Internacional de Ciencias del Deporte, XV (55), 88101. https://doi.org/10.5232/ricyde2019.05506

Martín-Domínguez, D. \& Rodríguez-Sánchez, S. (2010). Psicomotricidad: ¿Qué formación dicen poseer los profesores de educación infantil y educación especial de la provincia de Huelva? Revista Electrónica Interuniversitaria de Formación del Profesorado, 13(4), 295-305. https://doi.org/10.6018/ reifop.21.1.295271

Martínez-Martínez, F. D. \& González-Hernández, J. (2018). Práctica de actividad física, conducta prosocial y autoconcepto en adolescentes: Conexiones en el contexto escolar. Electronic Journal of Research in Educational Psychology, 16(46), 555577. https://doi.org/10.25115/ejrep.v16i46.2235

Mas, M., Jiménez, L. \& Riera, C. (2018). Systematization of the psychomotor activity and cognitive development. Psicología Educativa, 24(1), 38-41. https://doi.org/10.5093/psed2018a5

Menéndez-Santurio, J. I. (2018). El modelo de la responsabilidad personal y social en Educación Física: Perspectivas en la investigación actual. In E. López-Meneses, D. Cobos-Sanchiz, A. H. Martín-Padilla, L. Molina-García \& A. Jaén-Martínez (Eds.), Experiencias pedagógicas e innovación educativa: Aportaciones desde la praxis docente e investigadora, 2018, ISBN 978-8417219-78-9, págs. 38-50 (pp. 38-50). Madrid: Octaedro. https:/ /doi.org/10.12804/tj9789587840186

Mérida-Serrano, R., de los Ángeles Olivares-García, M. \& GonzalezAlfaya, M. E. (2018). Discovering the world through the body in the childhood. The importance of materials in the child psychomotricity. Retos-Nuevas Tendencias En Educacion Fisica Deporte y Recreacion, (34), 329-336.

Ministerio de Educación y Ciencia del Gobierno de España. (2007, 30/10/). Real Decreto 1393/2007, de 29 de octubre, por el que se establece la ordenación de las enseñanzas universitarias oficiales. Boletín Oficial del Estado, 1-28. https://doi.org/10.2307/ j.ctt22nmcxk.10

Ministerio de Educación y Ciencia del Gobierno de España. (2008). Orden ECI/3960/2007, de 19 de diciembre, por la que se establece el currículo y se regula la ordenación de la educación infantil. Boletín Oficial Del Estado, 1016-1036. https://doi.org/10.29156/ v4.i2/5

Missana, M., Rajhans, P., Atkinson, A. P. \& Grossmann, T. (2014). Discrimination of fearful and happy body postures in 8-monthold infants: An event-related potential study. Frontiers in Human Neuroscience, 8 (july). https://doi.org/10.3389/ fnhum.2014.00531

Monzonís-Martínez, N. (2019). La Educación Física como elemento de mejora de la competencia social y ciudadana. Investigación acción en torno a la aplicación de un programa basado en la prevención y resolución de conflictos desde el área de educación física. Apunts: Educación Física y Deportes, (135), 138-138. https://doi.org/10.5672/apunts.2014-0983.es.(2013/4).114.02

Mora-Teruel, F. (2014). Neuroeducación. Madrid: Alianza.

Nilsen, A. K. O., Anderssen, S. A., Resaland, G. K., Johannessen, K., Ylvisaaker, E. \& Aadland, E. (2019). Boys, older children, and highly active children benefit most from the preschool arena regarding moderate-to-vigorous physical activity: A crosssectional study of norwegian preschoolers. Preventive Medicine Reports, 14, $100837 . \quad$ https://doi.org/10.1016/ j.pmedr.2019.100837

Pellicer-Royo, I., López-González, L., Mateu-Serra, M., MestresPastor, L., Meritxell, M. H. \& Ruiz-Omeñaca, J. V. (2015). NeuroEF. La revolución de la Educación Física desde la Neurociencia. Barcelona, España: Editorial INDE.

Pérez González, M. C. (2011). Psicomotricidad: etapas en la elaboración del esquema corporal en educación infantil. Pedagogía Magna, (11), 360-365.

Piaget, J. (1975). El nacimiento de la inteligencia en el niño. Madrid: Aguilar.

Picq L. \& Vayer P. (1977). Educación psicomotriz y retraso mental. Barcelona: Científico-Médica. [Versión original: 1969].

Pierre, P. (2018). Une pédagogie des compétences motrices. Acción Motriz, (20), 89-96.

Pons, R. \& Arufe, V. (2016). Análisis descriptivo de las sesiones e instalaciones de psicomotricidad en el aula de educación infantil. Sportis Scientific Technical Journal, 2(1), 125-146. https:// doi.org/10.17979/sportis.2016.2.1.1445

Prat, Q., Camerino, O., Castañer, M., Andueza, J. \& Puigarnau, S. (2019). El modelo pedagógico de responsabilidad personal y social como motor de innovación en educación física. Apunts Educación Física y Deportes, (136), 83-99. https://doi.org/10.5672/ apunts.2014-0983.es.(2019/2).136.06

Rodríguez, M. T., Gómez, I. M., Prieto-Ayuso, A. \& Gil-Madrona, P. (2017). La educación psicomotriz en su contribución al desarrollo del lenguaje en niños que presentan necesidades específicas de apoyo educativo. Revista de Investigación en Logopedia, 7(1), 89-106. https://doi.org/10.5209/rlog.58734

Sampaio, J. O., De David, A. C., Filho, L. C. \& Húngaro, E. M. (2017). Bodily practice as an expression of children's imagination while playing: A historical-cultural psychology perspective. Movimento, 23(4), 1447-1458. doi:10.22456/1982-8918.72972

Secchi, J. D., García, G. C. \& Arcuri, C. R. (2016). ¿Evaluar la condición física en la escuela? Conceptos y discusiones planteadas en el ámbito de la educación física y la ciencia. Enfoques, 28(1), 6792. https://doi.org/10.28997/ruefd.v0i11.1

Silva, R., Abilleira, M. \& Gradín, M. (2018). Educación emocional y expresión corporal: Tratamiento integral en educación infantil. Trances. Revista de Transmisión del Conocimiento Educativo y de la Salud, 10(2), 121-136. https://doi.org/10.12795/ ie.2018.i89.04

Sugrañes-Justafré, E. \& Ángel-Ferrer, M. A. (2007). La educación psicomotriz (3-8 años): Cuerpo, movimiento, percepción, afectividad: Una propuesta teórica ( $1^{\mathrm{a}}$ ed.). Barcelona: Graó.

Teixeira Héctor José, Arufe-Giráldez, V., Abelairas-Gómez, C. \& Barcala-Furelos, R. (2015). The influence of a structured physical education plan on preschool children's psychomotor development profiles. Australasian Journal of Early Childhood, 40(2), 6877. https://doi.org/10.1177/183693911504000209

Terry-Andrés, J. (2014). Análisis de la influencia de la metodología de la intervención psicomotriz sobre el desarrollo de las habilidades motrices en niños de 3 a 4 años. Universidad de Murcia. Murcia. https://doi.org/10.5514/rmac.v1.i2.27099

Viscarro-Tomás, I.; Antón-Rosera, M. \& Cañabete-Ortiz, D. (2012). Perfil y formación de los profesionales que realizan la práctica psicomotriz en la etapa de educación infantil. El caso de las comarcas de Tarragona. Educar, 48(2), 321-344. https:/doi.org/ 10.5565/rev/educar.28

Vygotsky, L.S. (1979). El desarrollo de los procesos psicológicos superiores. Barcelona: Crítica.

Wallon, H. (1969). Los orígenes del carácter en el niño. Buenos Aires: Lautaro. [Versión original: 1934]

World Health Organization. (2019). Guidelines on physical activity, Zub d sleep for children under 5 years of age. ganization. https://doi.org/10.26719/

005). Algunas consideraciones sobre la o en la adquisición de habilidades motrices sicología del Deporte, 14(1), 85-95. https:/ 8-84232013000200007 